\title{
Toxoplasma granuloma of brainstem: A rare case
}

\author{
Anurag Gupta, A. Raja, A. Mahadevan'1, S. K. Shankar'1 \\ Department of Neurosurgery, KMC, Manipal, 'Department of Neuropathology, NIMHANS, Bangalore, Karnataka, India
}

\begin{abstract}
Toxoplasmosis is a common opportunistic infection in patients with AIDS in whom it frequently presents as intracranial space-occupying lesions. In the immunocompetent patient the most common manifestation is as asymptomatic cervical lymphadenopathy which may be associated with vague systemic manifestations such as fever or myalgia. In very rare cases people with normal immunity may present with meningoencephalitis polymyositis or myocarditis. It is very rare to encounter a brainstem granuloma due to toxoplasma infection in such patients. We report a nonimmunocompromised man who presented with multiple cranial nerve palsies due to a brainstem lesion, which turned out to be a toxoplasma granuloma. He recovered completely after a four-week course of Pyrimethamine and Sulphadoxine. An extensive search of the literature failed to reveal any prior reports of a similar nature. This case is being reported because of its rarity and the complete recovery made by the patient.
\end{abstract}

Key words: Brainstem, cranial nerve palsies, immunocompetent, toxoplasma

Toxoplasmosis presenting as intracranial spaceoccupying lesions in immunocompromised patients is a common occurrence, being one of the most common opportunistic lesions in patients with the Acquired Immuno Deficiency Syndrome (AIDS). ${ }^{[1,2]}$ In immunocompetent patients it has been reported to present with lymphadenopathy and rarely with diffuse central nervous system (CNS) symptoms. ${ }^{[3]}$

We would like to present an interesting case of CNS toxoplasmosis presenting as multiple cranial nerve palsies in a non-immunocompromised patient.

\section{Case Report}

A 27-year-old tailor presented to our neurological colleagues with complaints of sudden onset of headache associated with left-sided weakness and vomiting. He had history of a similar episode one month earlier which had resolved. On examination he was fully conscious and oriented. Anisocoria was present with the right pupil being larger but reactive. There was crossed hemiplegia with left hemiparesis and involvement of the right V VII XI X cranial nerves, suggestive of an intra-axial lesion.

An urgent magnetic resonance (MR) imaging of the brain was done, which on T1 weighted images revealed a hypointense pontine lesion with extension into the midbrain. The lesion was heterogeneously enhancing with contrast with vague ring enhancement dorsally [Figure 1].

In view of his age and the radiological features of the lesion the possibility of a neoplasm was kept in mind. A thorough hematological and radiological evaluation was done and they were essentially normal. In view of progressive signs of raised intracranial tension it was decided to proceed with a microsurgical biopsy and decompression of the lesion.

He underwent microsurgical decompression and biopsy of the lesion via a right sub temporal transtentorial approach. The tentorium was divided posterior to the III nerve taking care to preserve the IV nerve. A grayish white, moderately vascular lesion was encountered and adequate decompression was carried out. A small Weck clip was left in the cavity and tissue sent for histopathological examination. Intraoperatively the lesion seemed to be a high-grade glioma.

To our great surprise the lesion was reported as inflammatory, with the possibility of toxoplasmosis to be considered. The tissue showed areas of dense microglial reaction admixed with foamy histiocytes [Figure 2A] separating the fiber tracts. Focal areas of active necrosis with nuclear debris were also seen [Figure 2B]. Some of the entrapped venules revealed fibrinoid necrosis and luminal thrombosis. Along the edge of the necrotic zone, foci of spotty calcification were seen in addition to a few ruptured but calcified toxoplasma cysts [Figure 2C]. The histological features were characteristic of the encephalitic stage of toxoplasma infection.

In view of the above diagnosis the patient was further worked up with regards to his immune status. He 


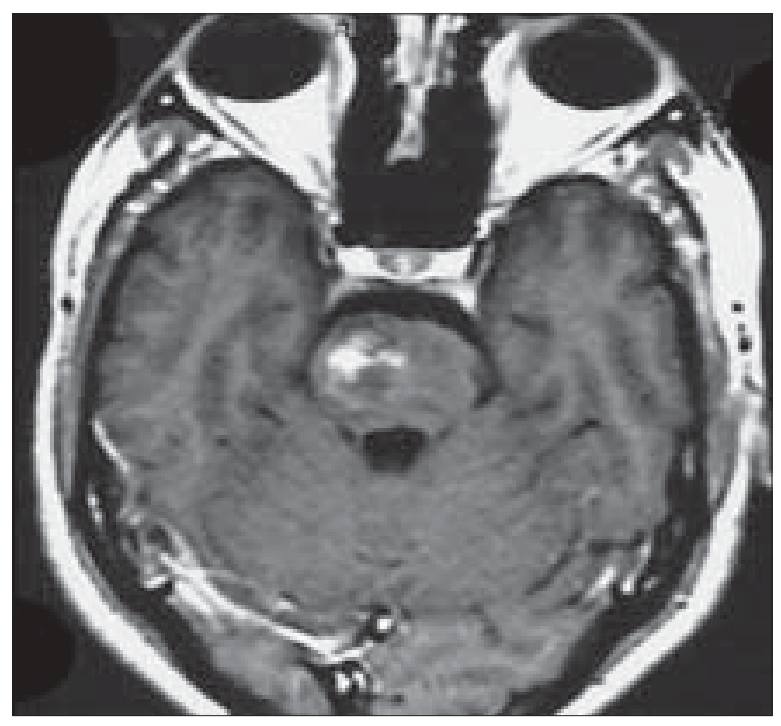

Figure 1: Pre op MRI showing hypointense lesion on T1 weighted images on the right side of the brainstem with a vague outline, the lesion is seen enhancing irregularly with contrast with a vague ring dorsally.

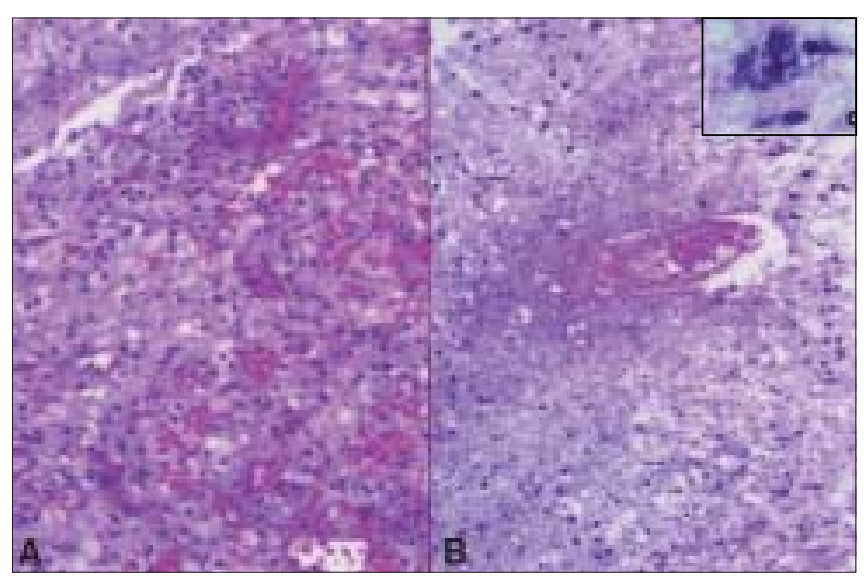

Figure 2: (A) Brain biopsy shows florid foamy histiocytic reaction admixed with lymphocytes and elongated microglial cells. (B) Areas of necrosis with nuclear debris and fibrinoid necrosis and thrombosis of venules in brain biopsy. (C) Ruptured toxoplasma cyst close to border of necrosis

did give a prior history of owning a cat. Two repeat ELISAs were negative for HIV. His total and differential counts were within normal limit with no evidence of any abnormal cells in the peripheral smear. A more thorough immunological workup was not feasible due to limitation of resources. His serial IgM and IgG titers for toxoplasma were also within the normal range.

As there was no evidence to suggest an underlying immune-deficient state and in view of the histopathological findings he was started on sulfadoxine and pyrimethamine combination for four weeks.

The patient showed marked improvement, with rapid improvement in his neurological status. At a six-month review he had made a complete recovery with no residual neurological deficits. Repeat MRI of the brain showed complete resolution of the lesion. Two years later he is doing extremely well with no neurological sequel and no fresh problems.

\section{Discussion}

Toxoplasmosis describes the clinical or pathological disease caused by T. gondii. T. gondii is a ubiquitous obligate intracellular protozoan. The members of the family Felidae (cats) are the only known definitive host of this organism. The main mode of transmission to man is either by ingestion of cyst or the oocyst or transplacental. Other less common reported modes are via a blood transfusion, organ transplantation and laboratory accidents. ${ }^{[3]}$

T. gondii has three forms: tachyzoites, bradyzoites and oocysts. The tachyzoites are the form seen in human tissues, they are oval or crescent-shaped, pointed at one end and oval at the other. They measure about $3 \times 7$ microns and stain well with either Wright or Geimsa stains. ${ }^{[4]}$

The clinical manifestation of toxoplasmosis can be divided into that seen in immunocompromised patients, that seen in immunocompetent patients and congenital.

In the immunocompetent patients only about $10-20 \%$ of all acute infections are symptomatic, the most common manifestation being asymptomatic cervical lymphadenopathy. This may be associated with vague systemic complaints such as fever or myalgia, they may also show signs of chorioretinitis. ${ }^{[3]}$ In very rare cases such patients may also present with meningoencephalitis, polymyosistis or myocarditis. ${ }^{[5]}$

On the other hand it is the most common pathological opportunistic infection and one of the commonest causes of intracranial space occupying lesions in patients with AIDS. Toxoplasma usually produces a diffuse or focal encephalopathy and is localized frequently to the cerebrum. Brainstem involvement is rare even in such patients and is usually associated with multiple cerebral lesions. ${ }^{[2,6]}$

The diagnosis of toxoplasma depends on either isolation of the organism, demonstration of the tachyzoites in the tissue by histology or by positive plasma serology. Diagnosis may also be made based upon the imaging features and response to therapy in AIDS patients. ${ }^{[1,2]}$

The lesions typically show up as multiple diffuse lesions with ring-like or solid enhancement on computed tomography (CT) of the brain. On MRI these lesions appear hypointense on T1 weighted images with up to $70 \%$ showing ring enhancement with contrast. The characteristic sign of CNS toxoplasmosis is the asymmetric target sign, which is detectable on both CT scans and MRI. This asymmetric target sign represents a ring-enhancing abscess, which contains other similar 
ring-enhancing abscesses as well as similarly enhancing, eccentric nodules in the abscess cavity. ${ }^{[7,8]}$

The treatment of choice for toxoplasma is a combination of Pyrimethamine $(1 \mathrm{mg} / \mathrm{kg} /$ day) and Sulfadiazine $(50 \mathrm{mg} / \mathrm{kg} \mathrm{q} 12 \mathrm{~h}$ or $6 \mathrm{~h}$ ) for four to six weeks or two weeks beyond resolution of symptoms. ${ }^{[4]}$

We are reporting this case because of its rarity. Although two cases of multiple intracranial lesions in immunocompetent patients due to toxoplasma have been reported, ${ }^{[9]}$ to our knowledge and despite an extensive search of reported literature, no reports of such solitary presentations of toxoplasma in a healthy individual were found. The fact that the patient has made a total recovery following medical treatment and has returned to his work as a tailor makes it even more remarkable.

\section{References}

1. Liesenfeld O, Wong SY, Remington JS. Toxoplasmosis. In: Goldmann L, Bennett JC, editors. Cecil textbook of medicine. $22^{\text {nd }}$ ed. Philadelphia PA, USA: W.B. Saunders; 2004. p. 2088-91.

2. Daras M, Koppel BS, Samkoff L, Mare J. Brainstem toxoplasmosis in patients with acquired immunodeficiency syndrome. J Neuroimag $1994 ; 4: 85-90$
3. Montoya JG, Remington JS. Toxoplasma gondii. In: Mandell GL, Douglas RG, Bennett JE, Dolin R, editors. Principles and practice of infectious diseases. 5th ed. Philadelphia: Churchill Livingstone; 2000. p. 2858-88.

4. McLeod R, Remington JS. Toxoplasmosis. In: Gorbach S, Bartlett JG, Blacklow NR, editors. Infectious diseases $2^{\text {nd }}$ ed. Philadelphia PA, USA: WB Saunders; 1992. p. 1328-42.

5. Kasper LH. Toxoplasma infection. In: Kasper DL, Braunwald E, Fauci AS, Hauser SL, Longo D, editors. Harrison's principles of internal medicine 16th ed. New York: McGraw-Hill; 2005. p. 1243-8.

6. Kure K, Harris C, Morin LS, Dickson DW. Solitary midbrain toxoplasmosis and olivary hypertrophy in a patient with acquired immunodeficiency syndrome. Clin Neuropathol 1989;8:35-40

7. Chinn RJ, Wilkinson ID, Hall-Craggs MA, Paley MN, Miller RF, Kendall BE, \& al. Toxoplasmosis and primary central nervous system lymphoma in HIV infection: Diagnosis with MR spectroscopy. Radiology $1995 ; 197: 649-54$.

8. Miguel J, Champalimaud JL, Borges A, Branco G, Doroana M, Medina E. Cerebral toxoplasmosis in AIDS patients, CT and MRI images and differential diagnostic problems. Acta Med Port 1996;9:29-36.

9. Vastava PB, Pradhan S, Jha S, Prasad KN, Kumar S, Gupta RK. MRI features of toxoplasma encephalitis in the immunocompetent host: A report of two cases. Neuroradiology 2002;44:834-8.

Accepted on 12-03-2008

Source of Support: Nil, Conflict of Interest: None declared. 